\title{
ÖKONOMISIERUNG
}

\section{Gegen eine Politik der Incentives}

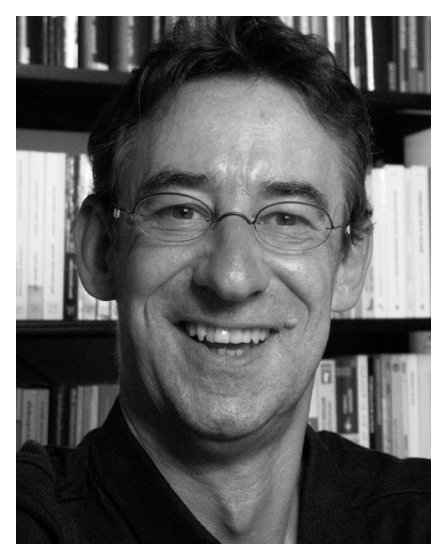

VON HARALD CHRISTA

Prof. Dr. Harald Christa ist Hochschullehrer für Sozialmanagement an der Evangelischen Hochschule Dresden sowie Berater und Gesellschafter der xit $\mathrm{GmbH}$ forschen.planen.beraten. in Nürnberg, Dresden und Berlin. harald.christa@ehs-dresden.de

\author{
Die Ökonomie hat inzwischen fast alle Lebensbereich \\ erfasst. Die Prinzipien einer wirtschaftlichen Denkweise \\ werden seit langem - durchaus mit Erfolg - auch auf \\ soziale Kontexte übertragen. Doch ist es auch ethisch \\ gerechtfertigt, wenn Strafgefangene gegen Bezahlung \\ eine Einzelzelle "upgraden « können - und ist eine solche \\ Gesellschaft auf Dauer von Menschen auszuhalten?
}

Ist es legitim, wenn eine Hilfsorganisation schwerst drogenabhängigen Frauen einen Geldbetrag dafür anbietet, dass sie sich sterilisieren lassen? Ist es akzeptabel, wenn eine Schulbehörde Jugendliche dafür entlohnt, dass sie regelmäßig zum Unterricht kommen, aktiv teilnehmen und gute Noten schreiben? Soll man Kindern fünf Euro für jedes gelesene Buch geben? Sollen wir Rauchern die erfolgreiche Teilnahme an Nichtraucherkursen vergüten? Und wenn ja: wie hoch? Solche und ähnliche Fragen diskutiert der politische Philosoph Michael J. Sandel mit Blick auf die in zunehmenden Maße zu beobachtenden Symptome einer Ökonomisierung von Lebenswelten.

Bei den genannten Maßnahmen handelt es sich um Interventionen, die funktioniert haben, welche also die intendierten Wirkungen (zumindest teilweise) erzielen konnten. Was spricht also dagegen, eine solche ökonomische Einflussnahme zu akzeptieren?

Auf den ersten (und vielleicht auch zweiten) Blick recht wenig. Vom Prinzip ist kaum etwas dagegen zu sagen, wenn Maßnahmen dazu führen, Gutes zu tun. Sterilisierte Frauen, die regelmäßig Heroin oder Crack konsumieren, gebären bei Erfolg der Maßnahme keine Kinder mehr, die bereits bei der Geburt drogenabhängig sind. Ehemals verhaltensauffällige Kinder und Jugendliche gehen dann regelmäßig zur Schule, verhalten sich angemessen im Unterricht, lesen gute Bücher und schreiben gute Noten. Raucher geben ihr Laster auf, leben länger und gesünder und ersparen der Versichertengemeinschaft viele Millionen.

Dass es aber trotzdem angezeigt ist, vermeintlich gute Absichten auf den Prüfstand zu stellen und sich zu fragen, was wir uns damit einhandeln, wenn wir wohlmeinend gesellschaftliche Ziele mit Geld verfolgen und quasi damit Marktprinzipien in soziale Kontexte einführen, zeigt uns der Autor anhand einer Diskussion einer Vielzahl von Beispielen, die in verschiedenen Ländern bereits Wirklichkeit geworden sind.

Michael J. Sandel geht bei seiner Analyse der Beispiele mit Bedacht vor. Er lässt Protagonisten wie Gegner entsprechender Maßnahmen und Programme zu Wort kommen und versucht durchaus, Verständnis für die (guten) Absichten zu zeigen, die hinter den jeweiligen ökonomischen Ideen stehen. Dies gilt beispielsweise auch für den Handel mit Verschmutzungsrechten, das gebührenpflichtige Schießen von Nashörnern in Afrika oder die gegen Geld zu vergebende Lizenz zum Kinderkriegen in übervölkerten Regionen.

Sandel räumt der Ideengeschichte einen breiten Raum ein. Der auf den amerikanischen Wirtschaftswissenschaftler Gary S. Becker zurückgehende Ansatz lautet, dass die Prinzipien der lange Zeit auf ihre klassischen Felder beschränk- 
ten ökonomischen Denkweise durchaus auch auf soziale Kontexte übertragen werden können. Dies gilt für die effiziente Verteilung von Gütern ebenso wie für erfolgreiche Anreize, mithin für eine effektive Steuerung. So entstand eine Ökonomie der Partnerwahl ebenso wie eine für die Genehmigung von Einwanderung.

Die Ökonomie hat ihr Feld seit fast fünfzig Jahren sukzessive erweitert und feiert die Erfolge ihrer Denkweise auch in gesellschaftlichen Bereichen wie Umweltverschmutzung, Justizvollzug und Straßenverkehr. Der globale Handel mit Emissionsrechten ist nahezu selbstverständlich geworden, in amerikanischen Gefängnissen können die Häftlinge gegen Gebühr eine Einzelzelle in »ruhiger Umgebung « zukaufen, in manchen amerikanischen Städten ist es möglich, gegen Gebühr auf einer gesonderten Überholspur zu fahren, wobei der Preis mit den Tageszeiten und das damit korrespondierende Verkehrsaufkommen variiert. Zweifellos darf dies als eine Maßnahme einer mikroökonomisch hochgebildeten Stadtverordnetenversammlung angesehen werden, aber entspricht sie dem Prinzip einer »fairen Gesellschaft«?

Eine wesentliche mikroökonomische Grundlage von »Preisen « ist dabei zu bedenken: Der Preis hat ökonomisch nur eine Funktion, er soll nicht zahlungsfähige oder zahlungswillige Marktteilnehmer vom Konsum ausschließen. Welche nicht intendierten Nebenwirkungen zweckrationaler Handlungen ergeben sich für eine Gesellschaft, in der die Bürgerinnen und Bürger über solche Schleichwege Stück für Stück an eine Ökonomisierung der Lebenswelt gewöhnt werden und damit Ungleichheit mit jedem Tag mehr im wahrsten Sinne des Wortes "erfahren"?

Manche der von Sandel aufgeführten Beispiele können in den Kontext von »Gerechtigkeit « oder »Gleichheit « eingeordnet werden und sollten damit Gegenstand eines breiten gesellschaftspolitischen Diskurses sein. Dieser muss klären, wie weit die Expansion des Konsums gehen kann und darf. Dieser muss zudem klären, ob es eine Gesellschaft in ihrem Empfinden für Gleichheit und Gerechtigkeit hinnehmen möchte, dass wohlhabendere Bürger es sich aufgrund ihrer Kaufkraft leisten können, auf der kostenpflichtigen Überholspur zu fah- ren, geschützte Tiere zu schießen, eine kürzere Zeit in der Warteschlange am Flughafen oder Museum zu verbringen, rascher als der gesetzlich Versicherte einen Arzttermin zu bekommen oder eine Leihmutter zu engagieren.

Andere Beispiele tangieren dagegen in direkter und möglicherweise brisanter Weise die Soziale Arbeit. Hier geht es nicht - wie eben beschrieben - um »Gebühren«, die Bevölkerungsgruppen mit größerem Geldbeutel einen Vorteil verschaffen oder Unpässlichkeiten ersparen können. Es geht vielmehr um »Anreize«, die ein politisch, sozial oder gesundheitlich erwünschtes Verhalten nach sich zu ziehen in der Lage sind und an die Stelle von etablierten (sozial-) pädagogischen Konzepten treten könnten.

Die Soziale Arbeit bekommt dann unangenehme »Konkurrenz« in Form der die von den Bürgern erhobenen zweckgebundenen Abgaben erhalten und dafür solche Stadtbewohner, die einen entsprechenden Beleg vorweisen können, vor Untaten verschonen. $\mathrm{Zu}$ Ende gedacht, könnte sich ein ganzer Sektor der Sozialen Arbeit der Gefahr ausgesetzt sehen, durch ein solchermaBen "einfaches « Instrument der Einflussnahme durch Geld ergänzt oder gar ersetzt zu werden. Schlimmstenfalls stellt sich heraus, dass monetäre Anreize auch noch erheblich kostengünstiger sind, als der Einsatz von Sozialpädagoginnen und Sozialpädagogen (inklusive der Aufwendungen für ihre Ausbildung an Fachhochschulen und Universitäten).

Sandel zeigt auf, wie sich das Wort »Anreize« über die Jahre sukzessive in Lehrbüchern, Medien und eben auch

\section{"Wie soll die Soziale Arbeit reagieren, wenn Bargeld für konformes Verhalten auf dem Schulhof erfolgreicher ist als Schulsozialarbeit?"}

eines neuen ökonomischen Problemlösungsdenkens, wenn sich zeigen sollte, dass Interventionen in Form von Bargeld für konformes Verhalten erfolgreicher sind als beispielsweise die Schulsozialarbeit oder die Erziehungshilfen. Was, wenn nachgewiesen werden kann, dass Jugendliche sich auf dem Schulhof friedlicher verhalten, wenn sie dafür in Euro entlohnt werden? Was, wenn sich ein Kind nach dem Versprechen, dafür Geld zu bekommen, sich nunmehr täglich den Hausaufgaben widmet, Prügeleien vermeidet und regelmäßig die Schule besucht? Was ist, wenn Eltern, die bislang die Erziehung ihrer Kinder grob vernachlässigt haben, nach der monetären Intervention nunmehr nahezu vorbildlich agieren? Wir könnten dies ausweiten auf die Straffälligenhilfe und Haftentlassenen adäquate Entlohnungen dafür anbieten, dass sie nicht mehr kriminell werden.

Der britische Autor Terry Pratchett hat dies bereits vor Jahren in seiner »Scheibenwelt-Satire" so formuliert, dass die Mörder und Diebe in der Großstadt in einer »Gilde « zusammengeschlossen sind, deren Mitglie- in der politischen Sprache ausgebreitet hat. Er verweist aber auch darauf, dass die Theorie der »Incentives « zwar auf motivationspsychologischen Grundlagen aufbauen kann, weiterhin jedoch strikt unabhängig von den Perspektiven von Moralphilosophie und politischer Philosophie behandelt wird. Die mittelbaren und unmittelbaren Folgen einer auf Anreize basierenden Denkund Vorgehensweise werden von der Ökonomie wie der Politik häufig nicht berücksichtigt.

In seiner Analyse der monetären Anreize kommt Sandel zum Schluss, dass wohlmeinende finanzielle Belohnungen für adäquates Verhalten dann dysfunktional werden, wenn dies in »Güterkategorien « verstanden wird und damit früher oder später eine »falsche Bewertung " des entsprechenden »Gutes" durch die Adressaten erfolgt. So könnte sich herausstellen, »dass der finanzielle Anreiz die intrinsische Motivation aushöhlt" und am Beispiel der Belohnung für Buchlektüre am Ende »weniger statt mehr gelesen wird «. Kinder können dann möglicherweise dazu verführt werden, »Bücherlesen als eine Art des Geld- 


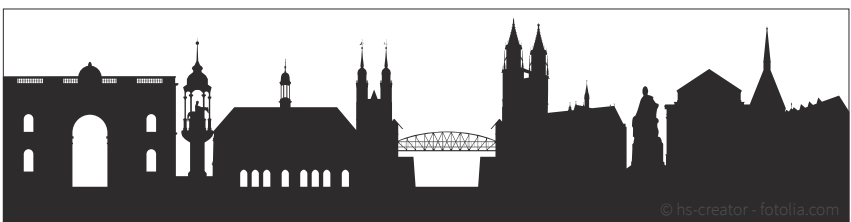

T. Pfeiffersche Stiftungen

Die Pfeifferschen Stiftungen in Magdeburg, mit über 1.000 Mitarbeitenden eine der größten diakonischen Komplexeinrichtungen Mitteldeutschlands, bieten ein umfassendes Leistungsspektrum für Menschen mit Unterstützungsbedarf, siehe auch pfeiffersche-stiftungen.de

Zu den Einrichtungen zählen unter anderem zwei Krankenhäuser, Wohnangebote für Menschen mit geistigen und mehrfachen Behinderungen, eine Werkstatt für behinderte Menschen, ambulante Pflegedienste und stationäre Altenhilfeangebote.

conQuaesso ${ }^{\circledR}$, die Personalberatung der contec $\mathrm{GmbH}$, sucht für die Werkstatt dieses Kunden für rund 490 Menschen mit Behinderung, zu der neben der Hauptwerkstatt auch eine Reha-Werkstatt für Menschen mit seelischer Beeinträchtigung zählt, zum nächstmöglichen Zeitpunkt eine

\section{Pädagogische Leitung WfbM / stellvertretende Bereichsleitung}

- Sie sind eine kommunikations- und organisationsstarke Persönlichkeit, qualifiziert in Pädagogik, Sozialpädagogik, Heilpädagogik oder vergleichbar, mit fundierten Kenntnissen und beruflicher Erfahrung in der Rehabilitationsarbeit.

- Sie können motivieren und begeistern, erfolgreich Teams und Konzepte entwickeln und kommunizieren gern mit den übrigen Professionen und Institutionen intern wie extern.

- Sie gehören einer christlichen Kirche im Sinne der ACK an.

Es erwartet Sie ein Arbeitsplatz mit ausgezeichneten Entwicklungsmöglichkeiten in einem hoch motivierten und gut eingespielten Team, umfangreichen Weiterbildungsangeboten und der Chance, eigene Ideen zu entwickeln und umzusetzen.

Ihre Bewerbung mit Angabe Ihrer Gehaltsvorstellung und des frühestmöglichen Eintrittstermins erbitten wir per Mail an conQuaesso ${ }^{\circledR}$ :

\section{leitung8211@conquaesso.de}

Für Informationen vorab:

Jonas Kessler, Telefon +49 (0) 16090584516 . Umfassende Vertraulichkeit sichern wir Ihnen selbstverständlich zu.<smiles>C1CC12CC2</smiles>

contec

conQuaesso

Personalberatung
conQuaesso $^{\circledR}$ - die Personalberatung der contec GmbH, Management- und Unternehmensberatung der Gesundheits- und Sozialwirtschaft. www.conquaesso.de

\section{Zukunftsweisender Lösungsansatz sozialer Problemlagen}
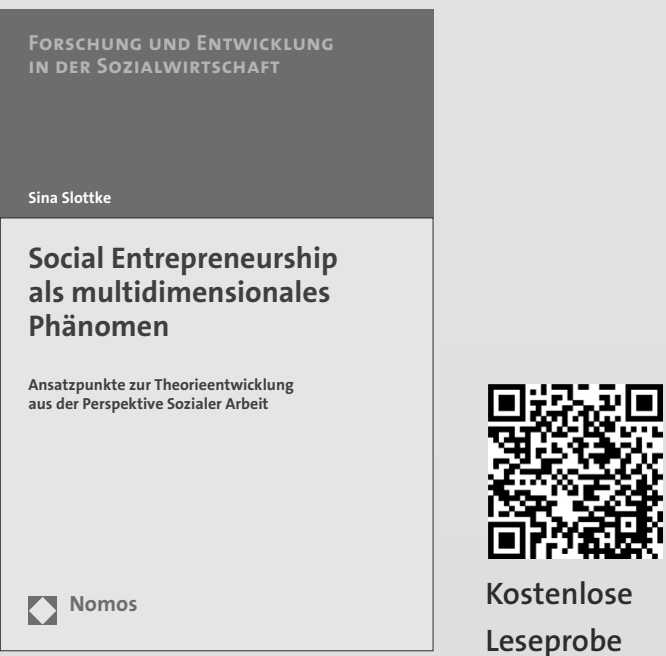

Kostenlose Leseprobe
Social Entrepreneurship als multidimensionales Phänomen

Ansatzpunkte zur Theorieentwicklung aus der Perspektive Sozialer Arbeit

Von Sina Slottke

2013, 104 S., brosch., 19,- $€$

ISBN 978-3-8487-0069-1

(Forschung und Entwicklung in der Sozialwirtschaft, Bd. 7)

Soziale Arbeit als Human Rights Profession erweitert die wirtschafts- und managementfokussierte Diskussion um Social Entrepreneurship als zukunftsweisendem Lösungsansatz sozialer Problemlagen um bisher unberücksichtigte inhaltliche und strukturelle Impulse und leistet somit einen Beitrag für die überfällige ethische Fundierung des populären Phänomens. 


WAS MAN
FÜR GELD
NICHT
KAUFEN
KANN
Die moralischen
Gren des Marktes
MICHAEL J.
SANDEL

Michael J. Sandel: Was man für Geld nicht kaufen kann. Die moralischen Grenzen des Marktes. Ullstein Taschenbuch Verlag, Berlin 2012. 304 Seiten. 9,99 Euro. ISBN 978-3-548-37526-7.

verdienens anzusehen ", und die »Liebe zum Lesen um seiner selbst willen « werde »verwässert, verdrängt oder korrumpiert«. Bezahlt man Menschen für beispielsweise eine gesunde Lebensweise, kann dies - so Sandel - »das Gegenteil dessen bewirken, was man eigentlich erreichen möchte, weil die Werte, die für die Erhaltung der Gesundheit wichtig sind, damit nicht kultiviert werden «. Es mag funktionieren, doch sind auf längere Sicht die Kollateralschäden möglicherweise enorm. In übertragener Form kann dies ebenso für die oben genannten Beispiele im Kontext von Straffreiheit oder einer vorbildlichen Erziehung von Kindern befürchtet werden.

Eine Politik der Incentives in sozialpädagogischen Kontexten führt dazu, dass Pädagogik abgeschafft und $\mathrm{Ma}$ nipulation eingeführt wird. Möchten wir in einer Gesellschaft leben, in der von den Menschen erwünschtes Sozialund Bildungsverhalten nur noch gegen Belohnung durch Geld an den Tag gelegt wird? Wo bleiben beispielsweise die Hilfe nach dem Unfall, die Solidarität bei Katastrophen, wenn gerade keine Anreize zur Hand sind? Wie kann der Wert von Bildung und Erziehung noch vermittelt werden, wenn die Menschen an eine pekuniäre Entlohnung gewohnt sind (und diese weiterhin erwarten)? Auch Freiwilligentätigkeit und Ehrenamt im Sozialen wären dann in Gefahr. $\mathrm{Zu}$ verweisen ist diesbezüglich auch auf
Studien, die klar belegen können, dass monetäre Anreize ehemals intrinsische Motivation verdrängen und damit in manchen Fällen eindeutig effizienzmindernd wirken können. Finanzielle Anreize beispielsweise beim Spendensammeln können bei Menschen, die vormals ehrenamtlich mit der Sammelbüchse unterwegs gewesen sind, dazu führen, dass sie nicht nur in gewisser Weise einen "Sinnverlust « erfahren, sondern weniger engagiert als vorher sind.

Normen des Marktes - so der Kommentar von Michael J. Sandel - »ersetzten moralisches und staatsbürgerliches Engagement oder dämpfte es zumindest «. Dies sollte auch Trägern der Sozialen Arbeit zu denken geben, die unreflektiert eine leistungsorientierte Vergütung einführen möchten. Der Aufwand, so die Ergebnisse der Studien, wäre insbesondere bei Konstellationen, in welchen die Mitarbeitenden ohnehin bereits eine intrinsische Motivation mitbringen, ungleich höher als der Ertrag.

\section{Resümee: \\ Wachsamkeit dringend geboten}

Die Einwürfe von Michael J. Sandel sind tiefsinnig, überzeugend und alarmierend. Er weist auf die Gefahren von Werte- und Kulturverlust eindringlich hin, wenn wir uns dort unkritisch einer ökonomischen Denkweise anschließen, wo sie nicht nur nichts zu suchen hat, sondern nachgerade kontraproduktiv wirken kann. Soweit uns bekannt, sind die beschriebenen Beispiele in Deutschland gegenwärtig weder erprobt noch angedacht. Andererseits sollten die Verantwortlichen in der Wohlfahrtspflege wachsam sein. Angesichts der schwachen Finanzlage von öffentlichen Haushalten, Krankenkassen und anderen tragenden Institutionen des Sozialstaates darf man jedoch skeptisch sein, ob die absolut lesenswerten Kommentare des amerikanischen Politikphilosophen bei der Mehrheit an zunehmend ethikferner entscheidenden Politikern Berücksichtigung finden wird.

Auch dürfte vielen Teilen unserer Bevölkerung die Lösung am nächsten sein, die ihren eigenen Geldbeutel verschont.

\section{Handbuch Controlling}

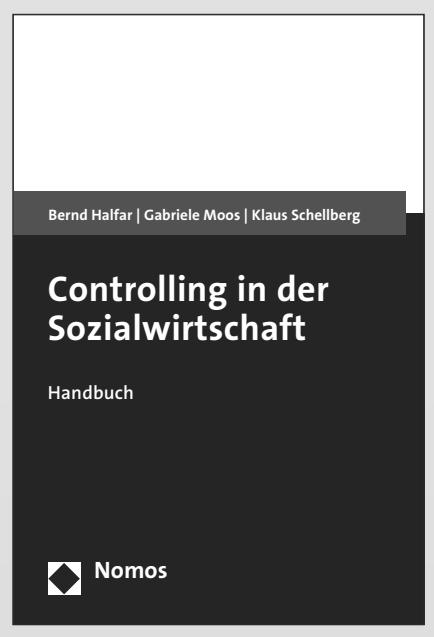

\section{Controlling in der Sozialwirtschaft}

Handbuch

Von Prof. Dr. Bernd Halfar und Prof. Dr. Gabriele Moos

2013, ca. 300 S., brosch., ca. 34,- $€$ ISBN 978-3-8329-6327-9

Erscheint ca. September 2013

Das Handbuch setzt an den Besonderheiten sozialer Dienstleistungsunternehmen an und entwickelt Vorschläge für ein praxistaugliches Controlling, das über das übliche Finanzcontrolling hinausreicht.

Der Leser findet thematisch geordnete Kennzahlenlisten und Anregungen, wie das Controlling die Produktivität sozialwirtschaftlicher Unternehmen analysieren kann.

www.nomos-shop.de/13315

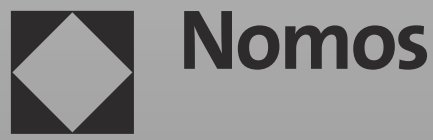

